\title{
The study of gas-liquid flow dynamics in the inclined-corrugated elements of cooling tower filler unit
}

\author{
Ilnur N. Madyshev ${ }^{1, *}$, Aliya I. Khafizova ${ }^{2}$, and Oksana S. Dmitrieva ${ }^{1}$ \\ ${ }^{1}$ Kazan National Research Technological University, 420015, Kazan, Karl Marx str., 68, Russia \\ ${ }^{2}$ Kazan State Power Engineering University, 420066, Kazan, Krasnoselskaya str., 51, Russia
}

\begin{abstract}
This paper deals with the studies of cooling tower, operated with the contactless evaporative cooling technology. The authors developed the cooling tower with a three-flow liquid cooling system. The authors conducted the numerical studies of gas-liquid flow dynamics in the inclined-corrugated elements of checker filling unit that allows to give us an idea of two-phase flow structure, its movement throughout the checker filling, as well as to assess the influence of mode parameters on the efficiency of collecting the liquid drops and the range of stable operation of device. The most effective operation of this device is at the pressure drop of $100 \mathrm{~Pa}$, while developing the average air flow rate in the element up to $3.2 \mathrm{~m} / \mathrm{s}$.
\end{abstract}

\section{Introduction}

Nowadays, the water resources in the world are under threat, that's why another problem is the pollution of these resources. A large amount of water is used for industrial needs, namely for the heat removal from the various types of process equipment. In order to reduce the water consumption for industrial needs, the enterprises use the circulating-water supply system, which significantly reduces the amount of water consumption. Water is cooled in the cooling towers mainly by atmospheric air within the volume of the cooling tower filler unit, which consists of checker filling layers, with mainly the countercurrent movement of phases [1-3].

Currently, the most cooling towers, used at the industrial enterprises, have significant physical and moral deterioration, while the amount of consumed energy resources is unreasonably overstated. In this regard, the relevant task for today is to modernize the water recycling systems, for example, to replace the old-fashioned filler units with new energy \resource-saving filler units. When manufacturing the new designs of filler units, a number of factors should be considered, namely: cooling efficiency, energy costs for execution of circulating-water system, steel consumption for design, simplicity to install and to replace with new contact elements, service life, and temperature requirements (temperature conditions are individual at each enterprise due to technological processes).

\footnotetext{
*Corresponding author: ilnyr 91@mail.ru
} 
We should not also forget about the biological contamination of water, caused by the availability of anaerobic bacteria and microorganisms in the network water. These organisms lead to the biofouling of the checker filling elements, thereby disrupting the structure of water and air flows, as well as they reduce the throughput capability, resulting in a decrease in the heat exchange, and, in some cases, cause the metal corrosion with its further destruction. The microorganisms enter the recycling water supply system with the atmospheric air, settle in the elements of checker filling and develop as a consequence of favorable conditions, such as: temperature increase, availability of dissolved oxygen, as well as other nutrients [4-7].

The most popular methods to control the pollution are the following: chemical, mechanical, hydro-mechanical, etc. The usage of chemical agents is widespread i.e. adding of them into the water medium, causing the death of microorganisms. However, this method has a number of disadvantages: one type of chemical agent destroys certain types of bacteria, but in order to destroy the others, it is necessary to use another type of chemical agent. Another disadvantage of chemical method is the fact that microorganisms adapt over time to the medium, containing the chemical agent and can develop in it. The purchase of chemical agents is expensive, since it is required to purchase sufficiently large amounts of them, as well as adding of them into the liquid requires additional material and physical investments. In this regard, it is necessary to develop the designs of filler units, considering all these requirements [8].

\section{Description of the device and its operation}

In order to eliminate the above shortcomings, an operation scheme of cooling tower with the contactless evaporative cooling technology was developed. This method of cooling the recycling water will significantly reduce the amount of chemical agents to be used.

The authors developed and proposed an original method of cooling the recycling water, which is shown schematically in Fig. 1. The layout diagram of cooling tower, within which the three-flow system of water and air takes place, is shown in Fig.1. The main elements of cooling tower are the following: filler unit 2, where water distribution and its cooling take place; water catchment basin 3, fan 1, which provides the movement of atmospheric air; water pump 4.

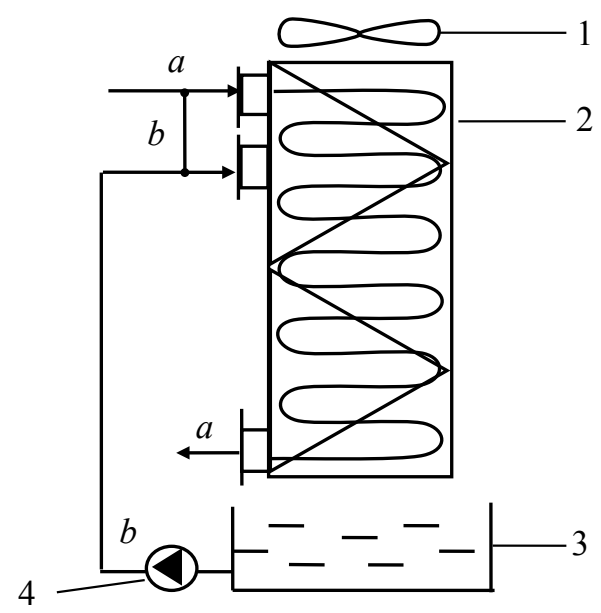

Fig. 1. The layout diagram of cooling tower with self-distribution of liquid throughout the filler unit: 1 - fan; 2 - filler unit; 3 - water catchment basin; 4 - water pump; $a$ - main hot water flow; $b$ irrigated water flow. 
The contactless evaporative cooling tower operates as follows: the hot water from the equipment, entering the device, is divided into two flows in such a way that the main flow (a) of recycling water enters the tubular radiator without contacting with the atmospheric air. The second water flow $(b)$ interacts with the air flow and is cooled by partial evaporation. This liquid flow acts as a refrigerant for the main flow, removing its heat through the pipe wall. Due to the fact that the main part of water is not in contact with the air, various impurities are not absorbed from it, therefore, the growth of microorganisms in the medium becomes impossible. The water flow $(b)$ consists of 5-6\% of hot water, supplied from the equipment and water in the catchment basin, which goes back to the input point of irrigated flow by means of water pump. The water level in the catchment basin is maintained due to the evaporation of liquid.

The cooling tower filler unit consists of several rows of checker filling units (Fig. 2). Each checker filling unit has one input point for water. The design of checker filling unit includes vertical wall on four sides, connected with each other, the area, in the cross section of which, forms a square. Within this space there are plates, made of thin metal 1, arranged in such a way that their profile forms a zigzag element. Each plate is located at an angle of $90^{\circ}$ against the other one. The plates are ribbon-flow contact elements with the horizontal corrugations of rounded profile. The availability of corrugations on the heat exchange surface creates a significant turbulence of moving liquid at a relatively low flow rate [9]. On the top of the plate there are 2 pipes in a row, located across the corrugations. The liquid flow moves in the recesses of plates and is directed from the top to bottom along a zigzag path. Some part of liquid falls down along the surface of wall of checker filling unit, the other part falls on the below located plate. There are the holes within the upper part of corrugated elements for the passage of atmospheric air. The air penetrates into the holes of plate, pushing the water drops out in different directions, thereby spraying the liquid throughout the checker filling and along the outer surface of pipes. The movement of air flow has a zigzag nature (it is shown by a dashed line in the figure): passing through the hole, goes around the pipe and moves up, turbulizing on the surface of steel plate.

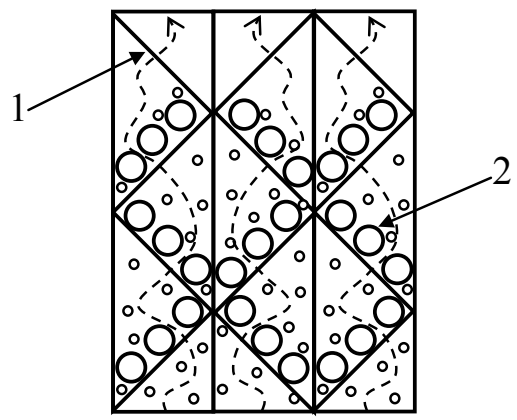

Fig. 2. The layout diagram of a row of checker fillings: 1 - corrugated plate; 2 - pipe.

There are several areas of interaction between the gas-liquid flows within the proposed checker filling unit: the film flow of liquid along the inclined corrugated plates and the horizontal pipes on the surfaces of plates, the passage of liquid jets through the holes within the plates, the collapse of these jets with the formation of large drops and the fragmentation of them during the subsequent interaction with the air flow and liquid film. As a result of spraying and collision of liquid drops with the surface of pipes, the heat exchange coefficients from the surface of pipes increase due to the evaporation of irrigating water. The studies by various authors $[10,11]$ show that the increase in the heat exchange coefficients is up to 30 times with the formation of a liquid film near the heated surface and spraying the water drops on this film. 
Due to such arrangement of plates, the self-distribution of liquid within the checker filling takes place, and the number of nozzles can be significantly reduced. Another advantage of this design is that each plate, located a stage above the previous one, acts as a drop collector, allowing us not to install additional separation devices [12].

\section{Description of the study and its results}

In order to determine the cooling efficiency of water, flowing through the corrugated plates, as well as to observe the trajectory of its movement, the authors carried out the numerical calculations. These numerical calculations were carried out by means of ANSYS Fluent software package, i.e. the simulation of liquid-gas interaction as exemplified by the "wateratmospheric air system". The flow cross-section area of one unit is $200 \times 229 \mathrm{~mm}$, the height of device is $440 \mathrm{~mm}$. The corrugated partitions are installed at an angle of $45^{\circ}$ to the horizon. There are 6 holes with the diameter of $20 \mathrm{~mm}$ within the upper circular segments of corrugations. The radius of circular segment of corrugation is equal to $20 \mathrm{~mm}$. The water was supplied into the contact device from a pipe with a diameter of $15 \mathrm{~mm}$, located on the side wall of checker filling.

In the course of studies, the liquid volume method was used, which allows to take into account the interaction of two immiscible phases. In the course of numerical simulations, the boundary conditions of pressure drops of air and water were set. The air pressure value within the upper part of device remained constant and was equal to $101325 \mathrm{~Pa}$. In the course of study, a non-stationary problem was solved. The calculations were suspended every time when the quasi-stationary regime was reached.

The results of studies show that with the pressure increase at the inlet of filler unit, the amount of liquid, entrained from the proposed contact device, increases. However, it should be noted that with the pressure increase, the average air flow rate also increases. Based on the graph (Fig. 3), showing the dependency of liquid entrainment on the average flow rate, it can be concluded that the device operates most effectively at the pressure drop of $100 \mathrm{~Pa}$. When the pressure drop is $100 \mathrm{~Pa}$, the liquid entrainment is equal on average to $15 \%$, while the flow rate, which can be developed by air within the element, can reach $3.2 \mathrm{~m} / \mathrm{s}$ that is quite high and shows a good capacity of the proposed device.

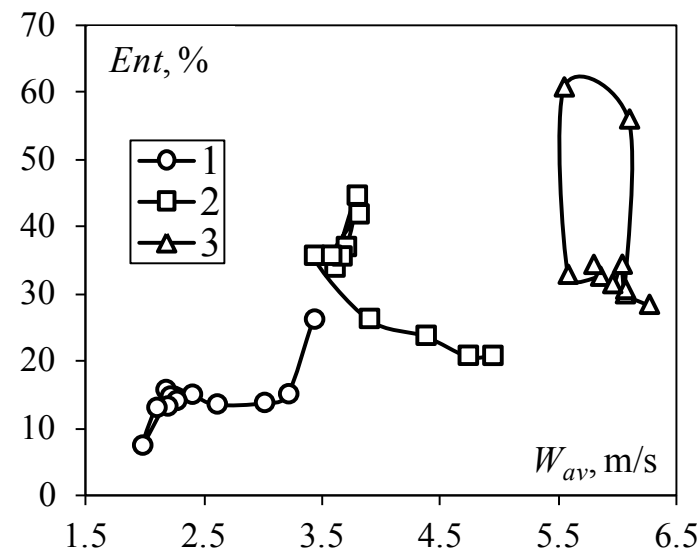

Fig. 3. The dependency of entrained liquid amount on the average air flow rate at different pressure drops in the contact device $\Delta P, \mathrm{~Pa}: 1-100 ; 2-200 ; 3-300$.

An increase in the ratio of mass flow rates of air and water leads to a decrease in the liquid entrainment from the device (Fig. 4). First of all, this is due to a decrease in the average rate of cooling air. 


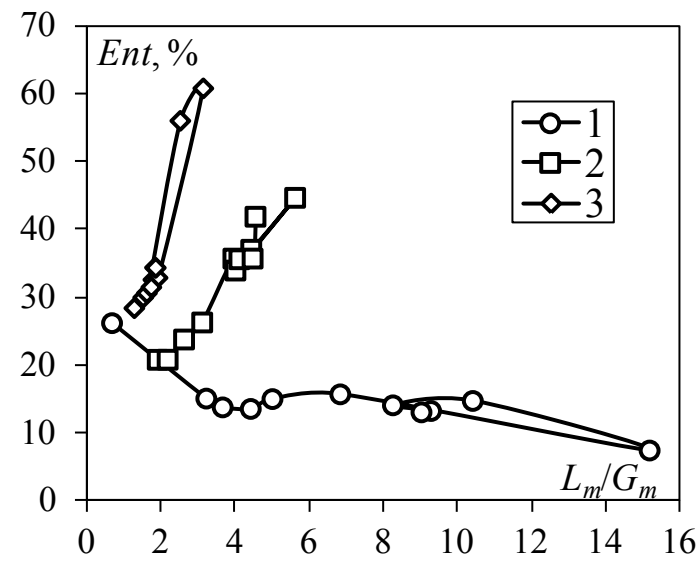

Fig. 4. The dependency of entrained liquid amount on the ratio of mass flow rates of air and water at different pressure drops in the contact device $\Delta P, \mathrm{~Pa}: 1-100 ; 2-200 ; 3-300$.

In the course of calculation, the graphical representation of liquid distribution within the checker filling can be seen; however, it should be noted that the upper partition prevents the liquid entrainment from the device, allowing the air to pass through the holes. The distribution of liquid within the device is uniform throughout the whole inside volume that indicates a large area of contact surface for the liquid and gas phases, and, consequently, the high efficiency of heat and mass exchange processes.

\section{Conclusion}

The conducted numerical studies show that with a low pressure drop in the proposed device, it is possible to reduce the entrainment of liquid drops. Moreover, the conducted studies allow to choose the most rational operation modes for the device while preserving the high separation efficiency. In addition, having studied the structure of two-phase gasliquid flow, it is possible to estimate the change in the liquid distribution within the device, depending on the inlet pressure. Thus, the most uniform distribution of liquid over the cross section of heat-mass exchange device is observed at a pressure drop of $100 \mathrm{~Pa}$.

The developed cooling tower filler units with self-distribution of liquid within the checker filling can provide a sufficiently high cooling efficiency of liquid without additional costs for the installation of drop collectors. If the influence of geometrical parameters of device on the cooling of liquid is further studies, then it will be possible to reduce the usage of chemical agents at the industrial enterprises significantly.

The study was conducted with funding from the grant of RF President № MK-417.2019.8.

\section{References}

1. X. Chen, F. Sun, Y. Chen, M. Gao, Appl. Therm. Eng., Novel method for improving the cooling performance of natural draft wet cooling towers, 147, 562-570 (2019)

2. A.B. Golovanchikov, N.A. Merentsov, V.A. Balashov, Chem. Pet. Eng., Modeling and analysis of a mechanical-draft cooling tower with wire packing and drip irrigation, 48, 9-10, 595-601 (2013)

3. E.V. Boev, S.P. Ivanov, V.G. Afanasenko, E.A. Nikolaev, Chem. Pet. Eng., Polymeric drop-film sprinklers for cooling towers, 45, 7, 454-459 (2009) 
4. A. Zaza, N.E. Laadel, E.G. Bennouna, Y.E. Hammami, M.T. Janan, Energy Procedia, Numerical study of the fouling effect on wet cooling towers designed to CSP plants, 157, 1230-1240 (2019)

5. N.P. Cheremisinoff, Pollution Control Handbook for Oil and Gas Engineering (Scrivener Publishing, 2016)

6. Y. Liu, W. Zhang, T. Sileika, R. Warta, N.P. Cianciotto, A.I. Packman, Biofouling, Disinfection of bacterial biofilms in pilot-scale cooling tower systems, 27(4), 393402 (2011)

7. I. Pagnier, M. Merchat, B. La Scola, Future Microbiology, Potentially pathogenic amoeba-associated microorganisms in cooling towers and their control, 4(5), 615629 (2009)

8. M.M. Zamaleev, V.I. Sharapov, News of heat supply industry, Measures to prevent the biological pollution of network water, 4, 43-47 (2015)

9. O.S. Dmitrieva, I.N. Madyshev, A.V. Dmitriev, J. Eng. Phys. Thermophy., Determination of the Heat and Mass Transfer Efficiency at the Contact Stage of a JetFilm Facility, 90(3), 651-656 (2017)

10. O.G. Martynenko etc., Reference book for the heat exchangers, 2 volumes T.1 (M.: Energoatomizdat, 1987)

11. A. Fraas, M. Ozisik, Calculation and design of heat exchangers (M.: Book upon demand, 2012)

12. V.G. Afanasenko, F.Sh. Khafizov, N.F. Khafizov, S.P. Ivanov, E.V. Boev, Chem. Pet. Eng., Development of designs for polymeric water traps in cooling towers using centrifugal separation forces, 43, 11-12, 653-656 (2007) 